Scientific Paper

\title{
Comparison of helical tomotherapy with multi-field intensity-modulated radiotherapy treatment plans using simultaneous integrated boost in high-risk prostate cancer
}

\author{
Hamit BAŞARAN ${ }^{a, b}$, Sibel KARACA ${ }^{b, c,}$, , Timur KOCA ${ }^{\mathrm{b}, \mathrm{c}}$, Yasemin Örs GÜNDOĞDU \\ ${ }^{a}$ Department of Radiation Oncology, Selçuk University Faculty of Medicine, Konya, Turkey \\ ${ }^{b}$ Sağllk Bilimleri University, Erzurum Regional Training and Research Hospital, Department of Radiation Oncology, \\ Erzurum, Turkey \\ ${ }^{c}$ Department of Radiation Oncology, Akdeniz University Faculty of Medicine, Antalya, Turkey \\ *E-mail address: sibeltuzlaci@gmail.com
}

\begin{abstract}
Purpose: The aim of this study is to compare the dosimetric results of Helical Tomotherapy (HT) and Multi-field IMRT treatment plans using a Simultaneous Integrated Boost (SIB) technique in the treatment of High-Risk Prostate Cancer (HRPCa) with pelvic nodal radiation.

Methods: Seventeen patients planned with HT and 7,8 and 9 fields IMRT were investigated. All plans were designed with the prescribed dose of $54.0 \mathrm{~Gy}$ to the $\mathrm{PTV}_{\mathrm{ln}}$ while simultaneously delivering $74.0 \mathrm{~Gy}$ to the $\mathrm{PTV}_{\mathrm{PS}}$ in 30 fractions. Dosimetric data of PTV and OARs were compared.

Results: HT gives a better CI and HI of PTV $\mathrm{PS}_{\mathrm{PS}}$ compared to multi-field IMRT plans. HT plans significantly improved target coverage (HT:0.95 vs multi-field IMRT: $0.52,0.49$ and 0.49 respectively, $\mathrm{p}<0.001$ ). Bladder mean dose(Gy) (HT: 45.6 vs multi-field IMRT: $53.6,53.3$ and 52.7 respectively, $\mathrm{p}=0.004)$ and $\mathrm{D}_{66 \%}(\mathrm{~Gy})$ dose (HT: 35.3 vs multi-field IMRT: 46.7, 47.0 and 44.9 respectively, $\mathrm{p}=0.006$ ) were lower in HT. But multi-field IMRT plans significantly reduced the rectum volume receiving more than $75 \mathrm{~Gy}$; ( $\mathrm{HT} \mathrm{V}_{75 \%}$ (\%) 2.7 vs multi-field IMRT $0.8,1.4$ and 0.9 respectively, $\mathrm{p}=0.008$ ). HT provided better sparing of the right and left femoral head receiving a mean dose. The penile bulb and small bowel doses were the highest in HT compared with multi-field IMRT.

Conclusions: HT achieved better dose distribution to target compared to multi-field IMRT. This study suggests HT as a reasonable option for the treatment of HRPCa patients.
\end{abstract}

Key words: prostate cancer; tomotherapy; intensity-modulated radiation therapy; simultaneous integrated boost.

\section{Introduction}

Prostate cancer $(\mathrm{PCa})$ is the most common type of cancer for men. Approximately one-tenth of all cancer deaths are related to PCa. ${ }^{1-2}$ Radiotherapy (RT) has an important role in the treatment of PCa. Using new technologies in RT, dose escalation is aimed at superior local control, better survival rates, and simultaneous improvement in quality of life. ${ }^{3-4}$ Owing to the Simultaneous Integrated Boosting (SIB) technique, different doses can be given simultaneously to different target volumes in a single fraction..$^{5}$ In this way, SIB makes it possible to simultaneously deliver different doses to the prostatic region and pelvic $\mathrm{LN}$ for PCa. ${ }^{6}$ Owing to the advances in technology (tomotherapy, IMRT, stereotactic RT, etc.) and improvements in planning methods, RT can be applied with a narrower limit $^{7}$ and less toxicity. As a result of technical developments in RT, conventional and three-dimensional conformal RT have been replaced by IMRT and tomotherapy techniques. IMRT is a method that enables better conformity in dose changes within the treatment area and allows to increase the tumor dose while the risk organ dose is decreased. Since PCa has a long-life expectancy, it is necessary to increase the effectiveness of the treatment. It is vital to increase the target volume by applying new RT techniques to patients and to protect organs at risk (OAR). The IMRT method used in PCa irradiation is a successful method for increasing the dose of the target by protecting the rectum and bladder and reducing genitourinary and gastrointestinal toxicity. In the linac-based IMRT technique, using the dynamic or static segments with 7-8-9 or more fields, the desired dose distribution is achieved in the target region while critical organs are protected. The recommended gantry angles and dose limits are not optimal, and each case should be handled individually according to anatomy. Recently, IMRT plans made with HT have become an attractive method in PCa radiation by creating higher conformity and homogeneity at target volume and superior plans in organ preservation.

(C) 2021 Hamit Başaran, Sibel Karaca, Timur Koca, Yasemin Örs Gündoğdu. This is an open access article licensed under the Creative Commons AttributionNonCommercial-NoDerivs License (http://creativecommons.org/licenses/by-nc-nd/4.0/). 
However, by using higher monitor units (MU) increases the time required for RT application. It is possible to eliminate this disadvantage of IMRT by using SIB. In the study of Cuccia et al. found that SIB technique provided homogeneous dose distribution to more than one tumor target and simultaneously gained saved up to $25 \%$ of the total treatment time. ${ }^{8}$ In an important review, Morikawa and Roach et al. ${ }^{9}$ highlighted the roles of all pelvis RT, volume identification and especially clinical target volume (CTV). In this study, we aimed to compare the plans made with the HT device and the linac based multi-fields IMRT plans (7, 8 and 9 fields) using the SIB technique for High-Risk Prostate Cancer (HRPCa).

\section{Methods}

\section{Patient selection and Simulation}

17 patients admitted to our clinic with the diagnosis of $\mathrm{HRPCa}$ were included in the study. As a result of the clinical evaluation, RT was planned for the pelvic lymph node (LN) region as well as the primary prostate and seminal vesicle, which have a high risk of pelvic relapse according to the Roachformula. According to our clinical protocol, bladder and rectum were emptied 30 minutes before the computerized tomography (CT) was taken. After emptying the bladder and rectum, patients were asked to drink $500 \mathrm{cc}$ water. Patients were applied $50 \mathrm{cc}$ IV contrast medium 20 minutes before the CT simulation. All patients were immobilized with a supine position with hip \& pelvic Vac-Lok and CIVCO "kneefix and feetfix" (CIVCO, Civco Medical Solutions, Kalona, IA, USA). CT images were taken with $3 \mathrm{~mm}$ slice thickness and were transmitted to the contouring workstation through Digital Imaging and Communications in Medicine (DICOM).

\section{Critical structure and target volume definitions}

The target and the critical organ volumes were outlined with a Focal (Elekta TM) workstation. CT images of patients were contoured by the radiation oncologist and reviewed by the 2 nd specialist according to the report of the International Commission on Radiation Units and Measurements (ICRU-83). The prostate and seminal vesicles were contoured as the target organ volume; the rectum, bladder, femur and penis bulb, sacral plexus and intestines are contoured as OAR volumes.

The Radiation Therapy Oncology Group (RTOG) prostate contouring atlas was used as a reference in the target volume drawing. RTOG GU Consensus pelvic LN CTV (CTV $\mathrm{Cn}_{\text {n }}$ volumes were used for pelvic $\mathrm{CTV}_{\ln }$ contours. ${ }^{10} \mathrm{~L} 5$ / $\mathrm{S} 1$ is contoured starting from the level of interspace (the level of the distal common iliac and proximal presacral lymph nodes) until femoral heads are removed. To create the $\mathrm{CTV}_{\mathrm{ln}}, 7 \mathrm{~mm}$ margin was given around the external and internal iliac vessels in each section. Furthermore, CTV prostate $\left(\mathrm{CTV}_{\mathrm{ps}}\right)$ was created by contacting the presacral lymph nodes from S1 to S3 with a thickness of about $10 \mathrm{~mm}$ from the front of the sacrum. According to the clinical stage of the patient, $\mathrm{CTV}_{\mathrm{ps}}$ was created by contouring the prostate \pm seminal vesicle previous to the planning target volume of prostate plus seminal vesicles (PTV $\mathrm{PS}_{\mathrm{PS}}$ and planning target volume of pelvic lymph node $\left(\mathrm{PTV}_{\mathrm{ln})}\right.$. A margin of $4 \mathrm{~mm}$ was given from the posterior and $7 \mathrm{~mm}$ from other directions added to $\mathrm{CTV}_{\mathrm{ps}}$ and a margin of $3 \mathrm{~mm}$ was given to $\mathrm{CTV}_{\ln } \cdot{ }^{11,12}$ The 3D images obtained were sent to the Hi-Art Tomotherapy and Linear Accelerator treatment planning system. The standard practice in our clinic is based on the Daily image guidance protocol using cone-beam CT for the patients during treatment. ${ }^{13}$ For this reason we reduced the planning margin for IGRT to provide the most conformal dose distributions in the study group.

\section{Treatment planning}

IMRT plans of 17 patients were made by $6 \mathrm{MV}$ photon energy and the data was uploaded to Synergy model, CMS, XIO (Elekta AB, Stockholm, Sweden) planning system. Three different step and shoot IMRT plans were made for each patient, from 7, 8 and 9 fields. Tomotherapy plans of 17 patients were made with HiArt HT planning system (Accuray Inc., Madison, USA) and 2.5$\mathrm{cm}$ field width, a pitch of 0.3 , and a modulation factor of 2-2.5 were used. 68 plans were obtained in total. The same dose limitations were used when making both device plans. The SIB technique was adopted in all planning. All plans were created to give a fraction dose of $2.46 \mathrm{~Gy}$ for $\mathrm{PTV}_{\mathrm{ps}}$ and a fraction dose of $1.8 \mathrm{~Gy}$ for $\mathrm{PTV}_{\ln }$ in 30 fractions. The prescribed dose was normalized to $98 \%$ volume. (The $100 \%$ of the PTV covered by $98 \%$ of the prescribed dose). Median dose $\left(\mathrm{D}_{50 \%}\right)$ was as close as possible to $54.7 \mathrm{~Gy}$ and $73.8 \mathrm{~Gy}$ for $\mathrm{PTV}_{\mathrm{ln}}$ and $\mathrm{PTV}_{\mathrm{ps}}$, respectively. As a clinical protocol, it was aimed to deliver at least 76.98 Gy and 80.95 Gy equivalent dose in 2 Gy fractions (EQD2) to $98 \%$ of $\mathrm{PTV}_{\mathrm{ps}}$ volume ( $\mathrm{D}_{98 \%}$ ) based on $\alpha / \beta$ ratio of $10 \mathrm{~Gy}$ and $3 \mathrm{~Gy}$ respectively. For OARs, the total dose was limited to $75.3 \mathrm{~Gy}$ for bladder and $75.8 \mathrm{~Gy}$ for rectum and small bowel. $\mathrm{D}_{\max }, \mathrm{D}_{\text {mean }}, \mathrm{D}_{\min }, \mathrm{D}_{98 \%}, \mathrm{D}_{2 \%}$, HI, CI, target coverage for PTV $_{\mathrm{ps}}$ and the median dose, $\mathrm{V}_{65 \%}, \mathrm{~V}_{70 \%}$ and $\mathrm{V}_{75 \%}$ doses for OARs obtained from the dose-volume histograms (DVH) of HT and linac-based IMRT plans were examined for all patients.

The resulting plans were compared and proposed by RTOG as evaluation criteria. ${ }^{14}$ The $\mathrm{CI}$ and $\mathrm{HI}, \mathrm{D}_{\max }, \mathrm{D}_{\text {mean }}, \mathrm{D}_{\min }$, of PTV $\mathrm{V}_{\mathrm{ps}}$ defined in report 83 of the International Commission on Radiation Units and Measurements (ICRU). ${ }^{15}$ All plans have been approved by the expert radiation oncologist and the OAR constraints were defined according to the Quantitative Analysis of Normal Tissue Effects in the Clinic (QUANTEC). ${ }^{16,17}$ 


\section{Homogeneity and conformity indices}

The concept of HI has been developed as an extension of the dosimetric analysis of treatment plans. Using HI was first proposed by RTOG in the routine evaluation of stereotactic RT (SRT) plans in 1993. HI was defined by RTOG = maximum isodose at target / reference isodose. If $\mathrm{HI} \leq 2$, the treatment was considered as following the protocol, if index is between 2 and 2.5 considered a minor violation, but an HI exceeding 2.5 is considered a major violation. However, the following formula for the calculation $\mathrm{HI}$ is more descriptive and widely used in the literature: ${ }^{18,19}$

$$
\mathrm{HI}=\frac{\begin{array}{c}
\text { minimum dose to } 2 \% \text { of the target volume }- \\
\text { dose to the } 98 \% \text { of the target volume }
\end{array}}{\text { prescribed dose }} \times 100
$$

$\mathrm{HI}$ is the ratio of the difference between maximum and minimum doses in the target volume so that low values of HI indicate a more homogeneous dose distribution in the volume. If $\mathrm{HI}$ is equal to or close to 0 , it indicates that the absorbed dose distribution is homogeneous. ${ }^{17}$

CI was developed by ICRU as part of the dosimetric analysis. CI is defined as a ratio between the volume covered by the reference isodose, which is $95 \%$ according to the ICRU.

CI RTOG $=$ Reference isodose volume / Target volume

When the CI value is equal to 1, ideal conformity is achieved. $\mathrm{CI}$ greater than 1 means that the irradiated volume is larger than the target volume and contains healthy tissues. If CI is less than 1 , only part of the target volume is irradiated. ${ }^{20}$

\section{Statistical analysis}

SPSS 18.0 for Windows (SPSS Inc., Chicago, IL) was used for statistical analysis of all data obtained from the study. Frequency tables for categorical variables and descriptive statistics including mean, standard deviation (SD), median, minimum and maximum for numerical variables are given. In the categorical comparisons between groups, cross-table statistics were given, and their significance levels were checked with the Chi-Square test. If the data was suitable for normal distribution, the results were given as "Average \pm SD" and "Median (MinimumMaximum)"; otherwise, p $<0.05$ value was considered significant.

\section{Results}

A total of 68 plans were created with the SIB technique. As a result of statistical analysis, obtained from HT and multi-field IMRT plans $\mathrm{D}_{\max }, \mathrm{D}_{\min }, \mathrm{D}_{\text {mean }}, \mathrm{D}_{98 \%}, \mathrm{D}_{2 \%}, \mathrm{HI}, \mathrm{CI}$ for $\mathrm{PTV}_{\mathrm{ln}}$ and PTV $_{\mathrm{ps}}$ are shown in Table $\mathbf{1 .}$

$D_{\max }(76.6 \mathrm{~Gy})$ obtained with the HT plan for $\mathrm{PTV}_{\mathrm{ps}}$ was lower than the other IMRT plans, but the difference was not statistically significant $(\mathrm{P}>0.05)$. When we look at the $\mathrm{D}_{98 \%}$ dose value of PTV $\mathrm{ps}_{\mathrm{ps}}$, HT and 7,8 and 9-field IMRT doses were 71.7 Gy, 68.2 Gy, 68.5 Gy and 68.4 Gy, respectively and the difference was significant in favor of HT $(\mathrm{P}=0.021)$. HI value of $\mathrm{PTV}_{\mathrm{ps}}$ found that $0.05(\mathrm{SD} \pm 0.02), 0.10$ ( $\left.\mathrm{SD} \pm 0.28\right), 0.09$ (SD \pm 0.09 ), and 0.09 (SD \pm 0.03 ) for HT and 7, 8 and 9-field IMRT plans respectively and the difference was significant in favor of HT (P < 0.05). The values of CI were found 1.47 (SD $\pm 0.45), 0.69(\mathrm{SD} \pm 0.23), 0.66(\mathrm{SD} \pm 0.04)$, and $0.62(\mathrm{SD} \pm 0.19)$ $(\mathrm{P}<0.001)$ for 4 plans respectively. The best coverage for $\mathrm{PTV}_{\mathrm{ps}}$ was HT among these 4 plans $(0.95(\mathrm{SD} \pm 0.01)),(\mathrm{P}<0.05)$. When the $\mathrm{D}_{98 \%}$ value of PTV $\mathrm{Pn}_{\mathrm{ln}}$ was examined, HT and 7,8 and 9field IMRT doses were 54.74 Gy (SD \pm 0.78$), 49.65$ Gy (SD \pm 4.66), $49.99 \mathrm{~Gy}(\mathrm{SD} \pm 3.63)$ and 50.39 Gy $(\mathrm{SD} \pm 4.04)$ respectively and the difference was significant in favor of $\mathrm{HT}(\mathrm{P}<0.05)$. The best coverage for $\mathrm{PTV}_{\mathrm{ps}}$ was HT among these plans $(0.92(\mathrm{SD} \pm 0.05))(\mathrm{P}<0.05)$.

An example image of HT and multi-field IMRT plans in PCa $\mathrm{RT}$ is presented in Figure 1. When the OAR doses were examined, HT and 7,8 and 9-field IMRT $\mathrm{D}_{\text {mean }}$ doses were 53.6 $\mathrm{Gy}(\mathrm{SD} \pm 6.16), 53.3 \mathrm{~Gy}(\mathrm{SD} \pm 7.68), 53.3 \mathrm{~Gy}(\mathrm{SD} \pm 7.68)$, and $52.7 \mathrm{~Gy}(\mathrm{SD} \pm 7.0)$ respectively for the bladder and the difference was significant in favor of HT $(\mathrm{P}<0.05)$. $\mathrm{D}_{66 \%}$ doses were $35.3 \mathrm{~Gy}(\mathrm{SD} \pm 11.79), 46.7 \mathrm{~Gy}(\mathrm{SD} \pm 9.39), 47.0 \mathrm{~Gy}(\mathrm{SD} \pm$ $12.53)$, and $44.9 \mathrm{~Gy}(\mathrm{SD} \pm 10.54)$ respectively for the bladder and the difference was significant in favor of HT $(\mathrm{P}<0.05)$.

\begin{tabular}{|c|c|c|c|c|c|c|c|c|c|}
\hline \multirow[b]{2}{*}{ Variable } & \multicolumn{2}{|c|}{ Helical Tomotherapy } & \multicolumn{2}{|c|}{ 7-field IMRT } & \multicolumn{2}{|c|}{ 8-field IMRT } & \multicolumn{2}{|c|}{ 9-field IMRT } & \multirow{2}{*}{ P-value } \\
\hline & mean & SD & mean & SD & mean & SD & mean & SD & \\
\hline PTV $_{\mathrm{ps}}$ & & & & & & & & & \\
\hline$D_{\max }(\mathbf{G y})$ & 76.6 & 3.70 & 78.9 & 4.08 & 78.0 & 4.16 & 77.3 & 3.37 & 0.323 \\
\hline$D_{\min }(G y)$ & 64.4 & 3.87 & 57.8 & 7.39 & 57.4 & 7.90 & 57.3 & 10.1 & 0.016 \\
\hline$D_{\text {mean }}(G y)$ & 73.8 & 3.34 & 72.4 & 3.20 & 72.3 & 3.46 & 72.3 & 3.32 & 0.904 \\
\hline $\mathbf{D}_{98 \%}(\mathbf{G y})$ & 71.7 & 3.65 & 68.2 & 3.74 & 68.4 & 3.97 & 68.5 & 3.85 & 0.021 \\
\hline $\mathbf{D}_{2 \%}(\mathbf{G y})$ & 75.1 & 3.39 & 75.6 & 3.39 & 75.3 & 3.62 & 74.9 & 3.24 & 0.920 \\
\hline CI & 1.47 & 0.45 & 0.69 & 0.23 & 0.66 & 0.12 & 0.62 & 0.19 & $p<0.001$ \\
\hline HI & 0.05 & 0.02 & 0.10 & 0.28 & 0.09 & 0.04 & 0.09 & 0.03 & $p<0.001$ \\
\hline Coverage & 0.95 & 0.01 & 0.52 & 0.16 & 0.49 & 0.09 & 0.49 & 0.09 & $p<0.001$ \\
\hline \multicolumn{10}{|l|}{ PTV $_{\text {In }}$} \\
\hline$D_{\max }(\mathbf{G y})$ & 72.5 & 3.89 & 71.6 & 3.51 & 71.8 & 4.5 & 71.44 & 3.92 & 0.961 \\
\hline$D_{\min }(\mathbf{G y})$ & 43.9 & 6.92 & 35.9 & 12.85 & 35.8 & 12.7 & 36.14 & 13.8 & 0.531 \\
\hline $\mathbf{D}_{98 \%}(\mathbf{G y})$ & 54.7 & 0.87 & 49.7 & 4.66 & 50.0 & 3.61 & 50.39 & 4.04 & 0.050 \\
\hline $\mathbf{D}_{2 \%}(\mathbf{G y})$ & 67.6 & 4.61 & 63.6 & 3.37 & 63.0 & 3.63 & 63.62 & 3.72 & 0.146 \\
\hline Coverage & 0.92 & 0.05 & 0.57 & 0.08 & 0.52 & 0.03 & 0.55 & 0.09 & $p<0.001$ \\
\hline
\end{tabular}

Table 1. HT and multi-field IMRT plans $D_{\max }, D_{\min }, D_{\text {mean }}, D_{98 \%}, D_{2 \%}$, HI, CI for PTV In $_{\text {and }}$ PTV 


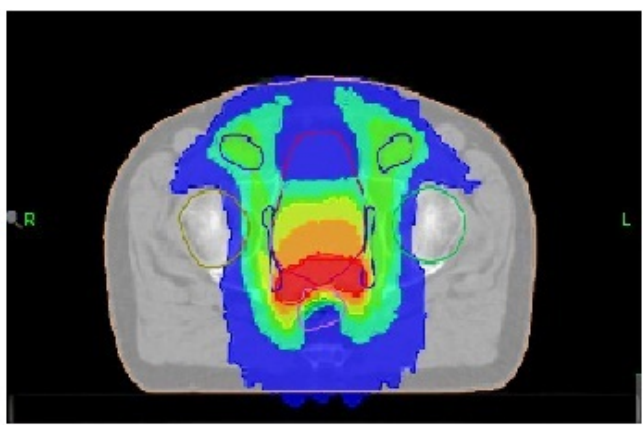

a.

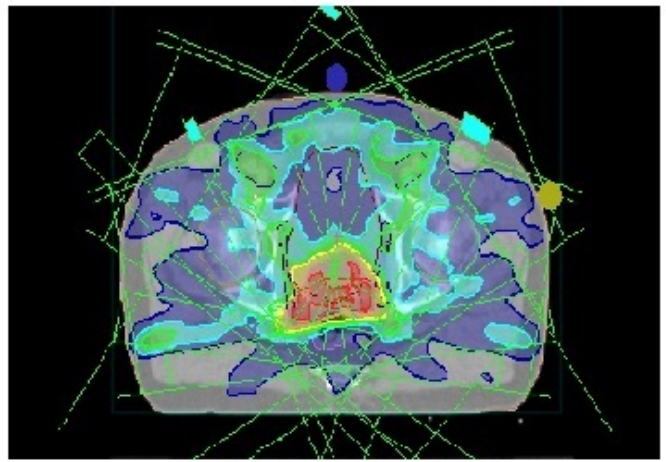

c.

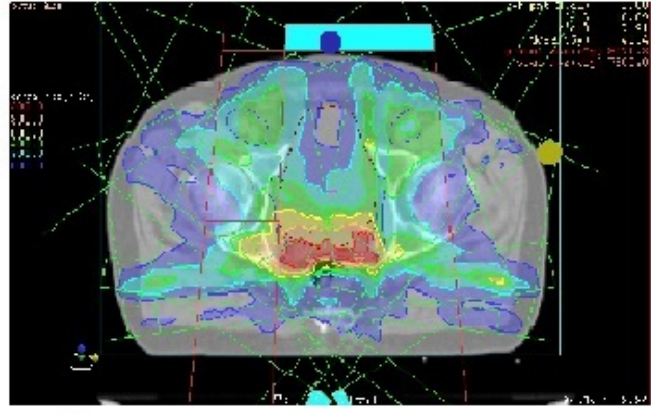

b.

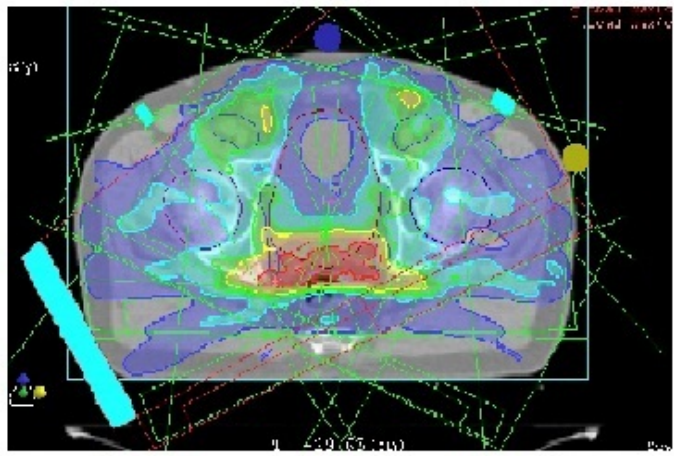

d.
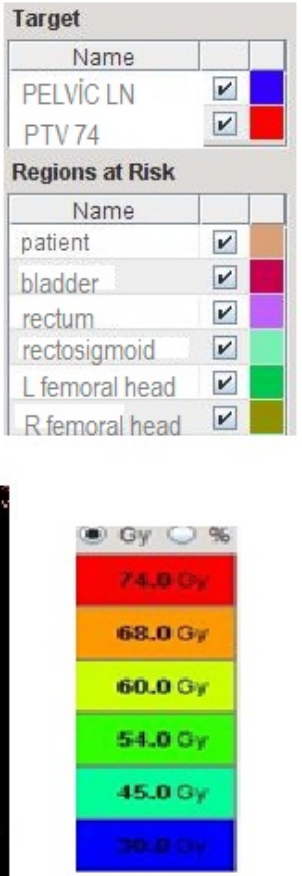

Figure 1. a. Axial view of dose distributions for helical tomotherapy. b. Axial view of dose distributions for 7-field IMRT. c. Axial view of dose distributions for 8-field IMRT. d. Axial view of dose distributions for 9-field IMRT.

$\mathrm{D}_{\text {mean }}$ doses were $41.9 \mathrm{~Gy}(\mathrm{SD} \pm 6.10), 44.5 \mathrm{~Gy}(\mathrm{SD} \pm 5.05)$, $45.5 \mathrm{~Gy}(\mathrm{SD} \pm 5.82)$, and $45.2 \mathrm{~Gy}(\mathrm{SD} \pm 5.00)$ respectively for rectum, and difference between them was not statistically significant $(\mathrm{P}>0.05)$. However, when we look at the results of the subgroup analysis, it was revealed that the HT was superior compared to the other three plans. $\mathrm{D}_{66 \%}$ doses were $75.8 \mathrm{~Gy}$ (SD $\pm 3.71), 76.2$ Gy (SD \pm 3.62$), 76.6 \mathrm{~Gy}(\mathrm{SD} \pm 4.58)$, and $75.3 \mathrm{~Gy}$ $(\mathrm{SD} \pm 3.51)$ for rectum, and the difference between them was not statistically significant $(\mathrm{P}>0.05)$. However, in subgroup analysis, HT was superior when compared with 7 and 8-field IMRT plans.

OAR doses of HT and multi-field IMRT treatment plans are shown in Table 2. We found that $\mathrm{D}_{\text {mean }}$ value was 15.9 Gy (SD $\pm 7.65)(\mathrm{P}<0.05)$ and 16.1 Gy $(\mathrm{SD} \pm 7.62)(\mathrm{P}<0.05)$ for the right and left femur, respectively. HT has proven to be significantly successful in protecting critical organs from the other three IMRT plans. Besides comparing the doses received by the sacral plexus values were determined according to the QUANTEC data, but there was no significant difference between the plans $(\mathrm{P}>0.05)$. The penile bulb and small bowel doses were the highest in HT.

\section{Discussion}

HRPCa is one of the most common tumors treated with IMRT. Therefore, this is a reasonable point for the evaluation of alternative IMRT techniques, such as HT simultaneously delivering technique or multi-field IMRT. In our study, we compared the DVH of the HT and 7,8 and 9-field IMRT in the treatment of HRPCa patients with pelvic RT. In the process of dosimetric comparison, $\mathrm{CI}$ and $\mathrm{HI}$ are found to be closely related to the complexity of target volumes as well as the delineation of volumes, the delivery equipment, the RT technology, and the optimization algorithm. ${ }^{21}$ In cancer treatment, more adaptation to the target volume and less damage to critical organs are the most important factors for choosing a radiation therapy modality. IMRT is successful in providing concave dose distribution for $\mathrm{PTV}_{\mathrm{ps}}$ wrapped with bladder and rectum. With advancing technology, the use of different planning methods of IMRT is increasing, especially in prostate cancers.

In the present study we used a 2.5 -cm field width, a pitch of 0.3 , and a modulation factor of 2-2.5 for HT planning. These parameters are similar to Skórska $\mathrm{M}$ and Piotrowski T's study of the tomotherapy parameter for prostate cancer. ${ }^{22}$ In our study showed that HT resulted in slightly superior conformity and homogeneity of PTV ${ }_{\mathrm{ps}}$ compared with 7, 8 and 9-field IMRT, respectively. HT significantly improved target coverage of PTV $_{\mathrm{ps}}$ compared to7, 8 and 9-field IMRT, respectively. The IMRT plans were similar in regard to rectum and bladder sparing, whereas the 9-field IMRT plan was superior to HT, 7 and 8-field IMRT plans. Most dosimetric comparison studies found that in treatment of prostate or prostate with seminal vesicles Modulated Arc Therapy provided equal or improved target coverage and OAR sparing compared to multi-filed IMRT. ${ }^{23}$ However, there is very little published data on dosimetric comparisons between HT and multi-field IMRT in the treatment of HRPCa. ${ }^{24}$ 
Table 2. Comparison of DVH parameters of OARs for HT and multi-field IMRT plans

\begin{tabular}{|c|c|c|c|c|c|c|c|c|c|}
\hline \multirow[b]{2}{*}{ Variable } & \multicolumn{2}{|c|}{ Helical Tomotherapy } & \multicolumn{2}{|c|}{ 7-field IMRT } & \multicolumn{2}{|c|}{ 8-field IMRT } & \multicolumn{2}{|c|}{ 9-field IMRT } & \multirow{2}{*}{ P-value } \\
\hline & Mean & SD & Mean & SD & Mean & SD & Mean & SD & \\
\hline \multicolumn{10}{|l|}{ Bladder } \\
\hline$D_{\max }(G y)$ & 75.3 & 3.44 & 76.7 & 4.03 & 76.3 & 3.92 & 75.5 & 3.88 & 0.660 \\
\hline $\mathbf{D}_{\text {mean }}(\mathbf{G y})$ & 45.6 & 7.88 & 53.6 & 6.16 & 53.3 & 7.68 & 52.7 & 7.00 & 0.004 \\
\hline $\mathbf{D}_{33 \%}(\mathbf{G y})$ & 55.9 & 7.39 & 60.2 & 7.46 & 61.1 & 6.11 & 60.4 & 5.29 & 0.086 \\
\hline$D_{66 \%}(G y)$ & 35.3 & 11.79 & 46.7 & 9.39 & 47.0 & 12.53 & 44.9 & 10.54 & 0.006 \\
\hline $\mathbf{D}_{99 \%}(\mathbf{G y})$ & 19.9 & 10.83 & 22.6 & 14.67 & 22.8 & 15.43 & 23.8 & 16.17 & 0.866 \\
\hline $\mathrm{V}_{65 \%}(\mathrm{ccc})$ & 27.3 & 15.77 & 38.5 & 23.72 & 40.0 & 28.64 & 35.7 & 22.27 & 0.415 \\
\hline $\mathbf{V}_{70 \%}(\mathbf{c c})$ & 16.1 & 14.16 & 21.3 & 20.38 & 21.2 & 19.78 & 19.7 & 18.84 & 0.876 \\
\hline $\mathrm{V}_{75 \%}(\mathrm{cc})$ & 5.4 & 6.86 & 4.1 & 5.99 & 3.4 & 4.09 & 2.6 & 3.41 & 0.351 \\
\hline \multicolumn{10}{|l|}{ Rectum } \\
\hline $\mathbf{D}_{\max }(\mathbf{G y})$ & 75.8 & 3.71 & 76.2 & 3.62 & 76.6 & 4.58 & 75.3 & 3.51 & 0.787 \\
\hline $\mathbf{D}_{\text {mean }}(\mathbf{G y})$ & 41.9 & 6.10 & 44.5 & 5.05 & 45.5 & 5.82 & 45.2 & 5.00 & 0.199 \\
\hline $\mathbf{D}_{\mathbf{3 3} \%}(\mathbf{G y})$ & 49.9 & 9.89 & 54.1 & 4.96 & 54.5 & 5.37 & 54.1 & 7.25 & 0.185 \\
\hline $\mathbf{D}_{66 \%}(\mathrm{~Gy})$ & 32.3 & 15.35 & 35.6 & 10.07 & 36.7 & 9.41 & 36.3 & 9.78 & 0.641 \\
\hline $\mathbf{D}_{99 \%}(\mathbf{G y})$ & 8.7 & 6.29 & 7.1 & 6.09 & 9.1 & 9.52 & 8.2 & 8.02 & 0.880 \\
\hline $\mathrm{V}_{50 \%}(\mathrm{cc})$ & 37.5 & 23.15 & 42.0 & 25.24 & 41.9 & 23.99 & 42.0 & 25.05 & 0.957 \\
\hline $\mathrm{V}_{60 \%}(\mathrm{cc})$ & 24.8 & 13.08 & 25.6 & 13.79 & 25.4 & 13.67 & 26.1 & 15.87 & 0.999 \\
\hline $\mathrm{V}_{65 \%}(\mathrm{cc})$ & 17.7 & 10.59 & 16.5 & 10.23 & 17.6 & 10.59 & 18.1 & 12.23 & 0.959 \\
\hline $\mathrm{V}_{70 \%}(\mathrm{cc})$ & 10.9 & 7.45 & 6.5 & 6.01 & 8.8 & 6.17 & 8.5 & 7.12 & 0.175 \\
\hline $\mathrm{V}_{75 \%}(\mathrm{cc})$ & 2.7 & 2.98 & 0.8 & 1.25 & 1.4 & 1.80 & 0.9 & 1.52 & 0.008 \\
\hline \multicolumn{10}{|c|}{ R. Femoral H. } \\
\hline$D_{\max }(\mathbf{G y})$ & 43.8 & 12.77 & 44.8 & 10.67 & 47.6 & 9.01 & 46.3 & 10.43 & 0.729 \\
\hline $\mathbf{D}_{\text {mean }}(\mathbf{G y})$ & 15.9 & 7.65 & 15.9 & 8.26 & 21.7 & 6.61 & 21.6 & 7.64 & 0.021 \\
\hline $\mathbf{D}_{33 \%}(\mathbf{G y})$ & 21.3 & 12.75 & 20.5 & 9.45 & 27.6 & 4.84 & 27.2 & 7.11 & 0.030 \\
\hline $\mathrm{D}_{66 \%}(\mathrm{~Gy})$ & 10.1 & 6.76 & 9.7 & 10.12 & 16.4 & 9.66 & 16.7 & 9.85 & 0.031 \\
\hline $\mathbf{D}_{99 \%}(\mathbf{G y})$ & 4.3 & 5.22 & 2.5 & 4.54 & 4.2 & 7.21 & 4.7 & 8.13 & 0.735 \\
\hline \multicolumn{10}{|c|}{ L. Femoral H. } \\
\hline$D_{\max }(\mathbf{G y})$ & 44.0 & 12.11 & 45.8 & 8.44 & 48.4 & 7.72 & 45.4 & 7.49 & 0.534 \\
\hline $\mathbf{D}_{\text {mean }}(\mathbf{G y})$ & 16.1 & 7.62 & 17.6 & 7.52 & 22.7 & 5.91 & 22.2 & 6.84 & 0.011 \\
\hline $\mathbf{D}_{\mathbf{3 3} \%}(\mathbf{G y})$ & 19.6 & 8.94 & 22.5 & 7.78 & 28.3 & 4.24 & 27.4 & 6.15 & 0.001 \\
\hline $\mathbf{D}_{66 \%}(\mathbf{G y})$ & 11.2 & 7.74 & 11.6 & 10.02 & 18.8 & 8.18 & 18.6 & 8.99 & 0.009 \\
\hline $\mathbf{D}_{99 \%}(\mathbf{G y})$ & 4.7 & 5.5 & 2.5 & 4.23 & 4.2 & 7.25 & 4.7 & 7.28 & 0.686 \\
\hline \multicolumn{10}{|l|}{ Small Bowel } \\
\hline $\mathbf{D}_{\max }(\mathbf{G y})$ & 60.3 & 19.39 & 63.2 & 21.00 & 62.6 & 20.28 & 60.6 & 20.48 & 0.979 \\
\hline $\mathbf{D}_{\text {mean }}(\mathbf{G y})$ & 10.74 & 9.53 & 9.1 & 8.25 & 8.9 & 8.70 & 9.3 & 9.25 & 0.951 \\
\hline $\mathbf{D}_{\mathbf{3 3} \%}(\mathbf{G y})$ & 15.2 & 16.39 & 8.2 & 11.58 & 8.0 & 11.56 & 8.4 & 12.11 & 0.351 \\
\hline $\mathbf{D}_{66 \%}(\mathbf{G y})$ & 9.3 & 11.37 & 4.8 & 7.90 & 4.8 & 7.80 & 5.1 & 8.49 & 0.431 \\
\hline $\mathrm{D}_{99 \%}(\mathrm{~Gy})$ & 4.9 & 7.56 & 0.3 & 0.37 & 0.4 & 0.65 & 0.4 & 0.54 & 0.006 \\
\hline \multicolumn{10}{|l|}{ Penile Bulb } \\
\hline$D_{\max }(\mathbf{G y})$ & 45.6 & 18.34 & 47.6 & 17.29 & 49.6 & 17.44 & 44.3 & 18.85 & 0.856 \\
\hline $\mathbf{D}_{\text {mean }}(\mathbf{G y})$ & 31.1 & 14.40 & 25.2 & 9.73 & 26.3 & 10.34 & 22.8 & 9.63 & 0.240 \\
\hline $\mathbf{D}_{33 \%}(\mathbf{G y})$ & 35.9 & 15.83 & 30.6 & 13.69 & 31.5 & 13.83 & 27.2 & 13.31 & 0.387 \\
\hline $\mathbf{D}_{66 \%}(\mathbf{G y})$ & 27.1 & 17.23 & 21.3 & 14.51 & 18.4 & 10.32 & 15.6 & 7.58 & 0.097 \\
\hline $\mathbf{D}_{99 \%}(\mathbf{G y})$ & 22.5 & 20.67 & 10.0 & 6.05 & 11.5 & 8.6 & 9.1 & 4.93 & 0.012 \\
\hline \multicolumn{10}{|l|}{ Sacral plexus } \\
\hline$D_{\max }(\mathbf{G y})$ & 37.3 & 26.79 & 39.7 & 29.8 & 37.9 & 28.15 & 37.1 & 27.43 & 0.996 \\
\hline $\mathbf{D}_{\text {mean }}(\mathbf{G y})$ & 11.6 & 10.27 & 22.1 & 20.5 & 20.3 & 18.48 & 19.4 & 18.07 & 0.512 \\
\hline $\mathbf{D}_{\mathbf{3 3} \%}(\mathbf{G y})$ & 10.5 & 10.46 & 24.2 & 23.75 & 22.4 & 21.78 & 20.3 & 20.22 & 0.195 \\
\hline $\mathbf{D}_{66 \%}(\mathbf{G y})$ & 5.7 & 4.77 & 15.9 & 19.25 & 14.9 & 16.94 & 14.2 & 16.62 & 0.191 \\
\hline $\mathbf{D}_{99 \%}(\mathbf{G y})$ & 4.1 & 2.83 & 11.3 & 15.06 & 6.2 & 8.60 & 8.1 & 11.58 & 0.279 \\
\hline
\end{tabular}

Rong Y et al. suggested that HT provided superior dose sparing for the rectum, whereas rapid arc showed superior dose sparing in the bladder in a patient treated with pelvic and a sequential boost to the prostate. ${ }^{25}$ Ishii $\mathrm{K}$. et al. compared treatment delivery efficiency among RapidArc, 7-field IMRT, and 9-field IMRT with hypofractionated SIB to the prostate. They concluded that treatment delivery efficiency is significantly higher for Rapid Arc. ${ }^{26}$

Davidson MT et al. quantified the differences in treatment delivery efficiency and dosimetry IMRT, Volumetric Modulated Arc Therapy (VMAT), and HT for prostate treatment. They found that HT resulted in a slightly higher integral dose and mean doses to the OAR, yielding a lower $\mathrm{D}_{\max }$ to all OAR examined. ${ }^{27}$ Pasquier et al. compared VMAT and HT in the treatment of HRPCa with pelvic nodal RT for the whole pelvis. They found that HT was able to provide a higher $\mathrm{D}_{98} \%$ than VMAT $(44.3 \pm 0.3$ Gy and $43.9 \pm 0.5 \mathrm{~Gy}$, respectively; $\mathrm{P}=0.032)$ and a lower $\mathrm{D}_{2} \%$ than VMAT $(47.3 \pm 0.3 \mathrm{~Gy}$ and 49.1 $\pm 0.7 \mathrm{~Gy}$, respectively; $\mathrm{P}=0.005$ ), leading to a better $\mathrm{HI} .^{28}$

Servagi-Vernat $\mathrm{S}$ et al. compared the dosimetric results of different techniques of dynamic IMRT in patients treated for pelvic cancer with nodal irradiation. Their results showed that despite of some dosimetric differences, HT and Rapid Arc provided very similar and highly conformal plans. Regarding OARs, Rapid Arc provided better pelvic bone sparing with a lower non-tumoral integral dose. ${ }^{29}$ 
Abu-Gheida I et al. reported 10-year outcomes for patients treated with IMRT with hypofractionated radiation therapy for prostate cancer in Cleveland Clinic. In median follow-up of 11.3 years, the 10-year clinical relapse-free survival rates were $95 \%$ and $72 \%$ for patients with low risk and high risk, respectively ( $\mathrm{P}$ $<0.0001)$. They concluded that high-dose moderately hypofractionated IMRT with daily image guidance for prostate cancer was found to deliver favorable 10-year oncologic outcomes with a low toxicity rate..$^{30}$

Widesott $\mathrm{L}$ et al. compared intensity-modulated proton therapy (IMPT) and HT treatment plans for HRPCa patients. They concluded that IMPT was expected to allow a small reduction in rectal toxicity, and a significant dosimetric gain was observed with IMPT, both in medium-dose and in low-dose range in all OARs. ${ }^{31}$

Barelkowski $\mathrm{T}$ et al. evaluated treatment outcomes for patients with HRPCa who were treated with dose-escalated radiation therapy with helical tomotherapy (HT) up to 84 Gy. They demonstrated that this scheme was both feasible and welltolerable. $^{32}$ According to the results of our study, the fractionation schedule appears to be acceptable with 74 Gy in 30 fractions at $2.46 \mathrm{~Gy}$ per fraction to $\mathrm{PTV}_{\mathrm{ps}}$. In comparison, HT allowed for dose escalation to the prostate with better sparing of the rectum than 7, 8, and 9-field IMRT. Beck $M$ et al. investigated dose-comparison in postoperative $\mathrm{PCa}$ patients using dose-intensified SIB in HT and found that this method was feasible and associated with favorable acute and late GU and GI toxicity rates. $^{33}$

The present study had some limitations that need to be mentioned. It was difficult to ensure that patients go into treatment fields in the same position at every treatment session. This could introduce some error and uncertainty to the measured values presented in this article. Another limitation is that this study includes only treatment planning parameters of different planning techniques. However, the effectiveness of the plans was not evaluated in terms of long-term clinical outcomes. Therefore, clinical investigations need to be done to evaluate likely correlations between dosimetric and clinical parameters for the defined techniques.

\section{Conclusion}

In conclusion, the dosimetric evaluation comparing HT to multifield IMRT underlines the potential of HT planning with SIB for HRPCa. There is a significant dosimetric advantage of HT over other multi-field IMRT techniques. This study suggests HT as a reasonable option for the treatment of HRPCa patients.

\section{References}

1. Siegel RL, Miller KD, Jemal A. Cancer statistics, 2020. CA Cancer J Clin. 2020;70(1):7-30. https://doi.org/ 10.3322/caac.21590

2. Bray F, Ferlay J, Soerjomataram I, et al. Global cancerstatistics 2018: GLOBOCAN estimates of incidence and mortality World wide for 36 cancers in 185 countries. CA Cancer J Clin. 2018;68(6):394-424. https://doi.org/10.3322/caac.21492. Erratum in: CA Cancer J Clin. 2020;70(4):313.

3. Kuban DA, Tucker SL, Dong L, et al. Long-termresults of the M. D. Anderson randomized dose-escalation trial for prostate cancer. Int J Radiat Oncol Biol Phys. 2008;70(1):67-74. https://doi.org/10.1016/j.ijrobp.2007.06.054

4. Dearnaley DP, Jovic G, Syndikus I, et al. Escalated-dose versus control-dose conformal radiotherapy for prostate cancer: long-term results from the MRC RT01 randomised controlled trial. Lancet Oncol. 2014;15(4):464-73. https://doi.org/ 10.1016/S14702045(14)70040-3

5. Cilla S, Deodato F, Digesù C, Macchia G, Picardi V, Ferro M, Sallustio G, De Spirito M, Piermattei A, Morganti AG. Assessing the feasibility of volumetric-modulated arc therapy using simultaneous integrated boost (SIB-VMAT): An analysis for complex head-neck, high-risk prostate and rectal cancer cases. Med Dosim. 2014 Spring;39(1):108-16. https://doi.org/ 10.1016/j.meddos.2013.11.001. Epub 2013 Dec 15. PMID: 24342167.

6. Saracino B, Petrongari MG, Marzi S, et al. Intensity-modulated pelvic radiation therapy and simultaneous integrated boost to the prostate area in patients with high-risk prostate cancer: a preliminary report of disease control. Cancer Med. 2014;3(5):1313-1321. https://doi.org/10.1002/cam4.278

7. Pacelli R, Caroprese M, Palma G, et al. Technologicalevolution of radiationtreatment: Implications for clinical applications. Semin Oncol. 2019;46(3):193-201. https://doi.org/10.1053/j.seminoncol.2019.07.004

8. Cuccia F, Mazzola R, Arcangeli S, et al. Moderate hypofractionated helical tomotherapy for localized prostate cancer: preliminary report of an observational prospective study. Tumori. 2019;105(6):516-523. https://doi.org/10.1177/0300891619867846

9. Morikawa LK, Roach M. Pelvic nodal radiotherapy in patients with unfavorable intermediate and high-risk prostate cancer: evidence, rationale, and future directions. Int J Radiat Oncol Biol Phys. 2011;80(1):6-16. https://doi.org/10.1016/j.ijrobp.2010.11.074

10. Lawton CA, Michalski J, El-Naqa I, et al. RTOG GU Radiation oncology specialists reachconsensus on pelvic lymph node volumes for high-risk prostate cancer. Int J Radiat Oncol Biol Phys. 2009;74(2):383-7. https://doi.org/10.1016/j.ijrobp.2008.08.002

11. Liang J, Wu Q, Yan D. The role of seminal vesicle motion in target margin assessment for online image-guided radiotherapy for prostate cancer. Int J Radiat Oncol Biol Phys. 2009;73(3):935-43. https://doi.org/10.1016/j.ijrobp.2008.10.019 
12. Ramiandrisoa F, Duvergé L, Castelli J, et al. Détermination des marges du volume cible anatomoclinique au volume cible prévisionnel pour la radiothérapie du cancer prostatique [Clinical to planning target volume margins in prostate cancer radiotherapy]. Cancer Radiother. 2016 Oct;20(6-7):629-39. French. https://doi.org/10.1016/j.canrad.2016.07.095

13. Piotrowski T, Kaczmarek K, Bajon T, et al. Evaluation of image-guidance strategies for prostate cancer. Technol Cancer Res Treat. 2014;13(6):583-91. https://doi.org/10.7785/tcrtexpress.2013.600258

14. Gay HA, Barthold HJ, O’Meara E, et al. Pelvic normal tissue contouring guidelines for radiationtherapy: A RadiationTherapy Oncology Group consensus panel atlas. Int J Radiat Oncol Biol Phys 2012;83:e353-e362. https://doi.org/10.1016/j.ijrobp.2012.01.023

15. International Commission on Radiation Units and Measurements Report 83. Prescribing, recording, and reporting photon-beam intensity-modulated radiation therapy (IMRT). J ICRU 2010;10(1). https://doi.org/10.1093/jicru/ndq002

16. Michalski JM, Gay H, Jackson A, Tucker SL, Deasy JO. Radiation dose-volume effects in radiation-induced rectal injury [published correction appears in Int J Radiat Oncol Biol Phys. 2019 Aug 1;104(5):1185]. Int J Radiat Oncol Biol Phys. 2010;76(3 Suppl):S123S129. https://doi.org/10.1016/j.ijrobp.2009.03.078

17. Wolff D, Stieler F, Welzel G, et al. Volumetric modulated arctherapy (VMAT) vs. serial tomotherapy, step-and-shoot IMRT and 3D conformal RT for treatment of prostate cancer. Radiother Oncol 2009; 93:226-233. https://doi.org/10.1016/j.radonc.2009.08.011

18. Shaw E, Kline R, Gillin M, et al. Radiation Therapy Oncology Group: Radiosurgery quality assurance guidelines. Int J Radiat Oncol Biol Phys. 1993;27:1231-1239. https://doi.org/10.1016/0360-3016(93)90548-a

19. Feuvret L, Noël G, Mazeron JJ, Bey P. Conformity index: a review. Int J Radiat Oncol Biol Phys. 2006;64(2):333-42. https://doi.org/ 10.1016/j.ijrobp.2005.09.028

20. Kataria T, Sharma K, Subramani V, et al. Homogeneity Index: An objective tool for assessment of conformal radiation treatments. J Med Phys. 2012;37(4):207-213. https://doi.org/10.4103/0971-6203.103606

21. Ning ZH, Mu JM, Jin JX, et al. Single arc volumetric-modulated arc therapy is sufficient for nasopharyngeal carcinoma: a dosimetric comparison with dual arc VMAT and dynamic MLC and step-and-shoot intensity-modulated radiotherapy. Radiat Oncol. $2013 ; 8: 237$. https://doi.org/10.1186/1748-717X-8-237

22. Skórska M, Piotrowski T. Optimization of treatment planning parameters used in tomotherapy for prostate cancer patients. Phys Med. 2013;29(3):273-85. https://doi.org/ 10.1016/j.ejmp.2012.03.007

23. Cao D, Holmes TW, Afghan MK, Shepard DM. Comparison of plan quality provided by intensity-modulated arc therapy and helical tomotherapy. Int J Radiat Oncol Biol Phys. 2007;69(1):240-50. https://doi.org/10.1016/j.jjrobp.2007.04.073

24. Tsai CL, Wu JK, Chao HL, et al. Treatment and dosimetric advantages between VMAT, IMRT, and helical tomotherapy in prostate cancer. Med Dosim. 2011;36(3):264-71. https://doi.org/10.1016/j.meddos.2010.05.001

25. Rong Y, Tang G, Welsh JS, et al. Helical tomotherapy versus single-arc intensity-modulated arctherapy: a collaborative dosimetric comparison between two institutions. Int J Radiat Oncol Biol Phys. 2011;81(1):284-96. https://doi.org/10.1016/j.ijrobp.2010.10.059

26. Ishii K, Ogino R, Okada W, et al. A dosimetric comparison of RapidArc and IMRT with hypofractionated simultaneous integrated boost to the prostate for treatment of prostate cancer. Br J Radiol. 2013;86(1030):20130199. https://doi.org/10.1259/bjr.20130199

27. Davidson MT, Blake SJ, Batchelar DL, et al. Assessingthe role of volumetricmodulatedarctherapy (VMAT) relative to IMRT and helical tomotherapy in the management of localized, locally advanced, and post-operative prostate cancer. Int J Radiat Oncol Biol Phys. 2011;80(5):1550-8. https://doi.org/10.1016/j.jirobp.2010.10.024

28. Pasquier D, Cavillon F, Lacornerie T, et al. A dosimetric comparison of tomotherapy and volumetric modulated arc therapy in the treatment of high-risk prostate cancer with pelvic nodal radiation therapy. Int J Radiat Oncol Biol Phys. 2013;85(2):549-554. https://doi.org/10.1016/j.ijrobp.2012.03.046

29. Servagi-Vernat S, Giraud P, Fenoglietto P, et al. Apport de la RCMI rotationnelle et de la tomothérapiehélicoïdaledans les cancers pelviens : étudedosimétrique prospective sur 51 patients [Impact of dynamic IMRT and tomotherapy in pelvic cancers: a prospective dosimetric study with 51 patients]. Cancer Radiother. 2014;18(2):111-118. https://doi.org/10.1016/j.canrad.2013.12.008

30. Abu-Gheida I, Reddy CA, Kotecha R, et al. Ten-Year Outcomes of Moderately Hypofractionated (70 Gy in 28 fractions) Intensity Modulated Radiation Therapy for Localized Prostate Cancer. Int J Radiat Oncol Biol Phys. 2019;104(2):325-333. https://doi.org/10.1016/j.ijrobp.2019.01.091

31. Widesott L, Pierelli A, Fiorino C, et al. Helical tomotherapy vs. intensity-modulated proton therapy for whole pelvis irradiation in high-risk prostate cancer patients: dosimetric, normal tissue complication probability, and generalized equivalent uniform dose analysis. Int J Radiat Oncol Biol Phys. 2011;80(5):1589-1600. https://doi.org/10.1016/j.ijrobp. 2010.10.005

32. Barelkowski T, Wust P, Kaul D, et al. Image-guided dose-escalated radiation therapy for localized prostate cancer with helical tomotherapy. Strahlenther Onkol. 2020;196(3):229-242. https://doi.org/10.1007/s00066-019-01562-2

33. Beck M, Wust P, Barelkowski T, et al. Risk adapted dose-intensified postoperative radiation therapy in prostate cancer patients using a simultaneous integrated boost technique applied with helical Tomotherapy. Radiat Oncol. 2017;12(1):125. https://doi.org/10.1186/s13014-017-0862-4 